\title{
Maxwell's equations and the vector nonlinear Schrödinger equation
}

\author{
Yijiang Chen \\ Optical Sciences Centre, Australian National University, Canberra ACT 2601, Australia \\ Javid Atai \\ Department of Electrical Engineering, MESA Research Institute, University of Twente, 7500 AE Enschede, The Netherlands
}

(Received 10 June 1996; revised manuscript received 28 August 1996)

\begin{abstract}
We examine similarities and fundamental differences between Maxwell's equations and the vector nonlinear Schrödinger equation (which is an approximation of the former) in describing a light evolution in a uniform nonlinear anisotropic medium. It is shown that in some cases, the solitary wave solutions to the nonlinear Schrödinger equation cannot be recovered from Maxwell's equations while in others the solitary wave solutions to Maxwell's equations are lost from the nonlinear Schrödinger equation through approximation (even in the limit under which the approximation is derived or valid), although there are cases where the solutions to the two sets of equations demonstrate only quantitative differences. The existence of novel classes of the hybrid vector solitary waves composed of three field components is also demonstrated and the bifurcation characteristics of the solitary wave states are analyzed. [S1063-651X(97)15303-X]
\end{abstract}

PACS number(s): 42.65.Tg, 42.65.Jx

\section{INTRODUCTION}

A light beam may propagate in a nonlinear medium without diffraction or dispersion when the diffraction contributing to the spread of the beam is balanced by the confinement resulting from the beam induced refractive index change in the material [1-3]. This study of solitary wave evolution in a uniform medium has aroused great interest recently, not only because of their observations in experiments [4-7] but also because of their diverse potential applications to ultrafast signal processing such as optical switching, computing, filtering, and beam splitting [7]. Over the extensive research reported so far, the theoretical and numerical analyses have mainly been based on the nonlinear Schrödinger equation, which normally involves one field component [8-13]. Indeed, an attempt was made to study the characteristics of the solitary wave solutions to the vector nonlinear Schrödinger equation (VNSE) with two field components, taking into account the polarization effect of the solitary waves [14]. In the presence of the two field components, VNSE for the nonlinear isotropic medium was proven to be integrable [14], supporting solitary wave solutions with a linear polarization oriented in an arbitrary direction. Recently, the study on VNSE was extended to higher-order solitary waves (i.e., stationary self-trapped beams) [15-20] and dynamic solitary waves $[21,22]$. It was shown that a proper superposition of the two field components of the first higher-order vector solitary wave [15] may lead to stationary propagation of the two orthogonally polarized parallel fundamental solitary waves [19].

Strictly speaking, light propagation in a medium obeys Maxwell's equations and VNSE is an approximation of Maxwell's equations. Naturally, a question remains as to whether VNSE yields a reliable approximation for portraying light evolution in a medium. In the nonlinear medium where nonlinearity results from electrostriction or $B=0$ in nonlinear polarization $\mathbf{P}_{\mathrm{NL}}=A\left(\mathbf{E} \cdot \mathbf{E}^{*}\right) \mathbf{E}+0.5 B(\mathbf{E} \cdot \mathbf{E}) \mathbf{E}^{*}[23]$, it has been shown that spatial solitary waves of Maxwell's equa- tions differ significantly from the classical solitary waves of the nonlinear Schrödinger equation unless the electric field is linearly polarized along a geometric axis of the beam intensity pattern [24]. In general the polarization state of a solitary wave in the nonlinear medium with $B=0$ changes continuously as the beam (dynamic solitary wave) propagates [24]. In such a nonlinear medium, Maxwell's equations were in fact shown to admit (not well behaved as shown in Fig. 2 of Ref. [25]) stationary solutions with the magnitude of the longitudinal field comparable to those of transverse fields that do not obey VNSE [25]. This difference between Maxwell's equations and VNSE for the case of $B=0$ is, however, not surprising as the approximation leading to VNSE is violated for these solutions [25], bearing in mind that VNSE is derived from Maxwell's equations under the limitation of small induced refractive index change or negligible longitudinal field in comparison with the transverse fields.

In this paper, we will examine differences and similarities between Maxwell's equations and VNSE for predicting the evolution patterns of solitary wave states trapped in a general uniform $(B \neq 0)$ nonlinear medium in the limit of small induced refractive index change (or small longitudinal field component) under which the VNSE is derived or valid. We will show that in some cases VNSE does give the solutions quantitatively different from those resulting from Maxwell's equations, whereas in others the solitary wave solutions to the two sets of equations demonstrate fundamental differences. Take the example of arbitrarily linearly polarized fundamental bright vector solitary waves with the two transverse field components being in phase predicted by VNSE [14]. It is found that this type of solitary wave does not obey Maxwell's equations, i.e., no arbitrarily linearly polarized fundamental vector solitary waves exist. On the other hand, for the two transverse field components being out of phase by $\pi / 2$ we find the existence of the fundamental hybrid (well behaved, i.e., one hump in each transverse field) TE-TM type vector solitary waves involving three field components; TE and TM components share almost the same contribution to 
the hybrid TE-TM solitary wave for infinitesimally small induced index change but the TM component tends to dominate with increasing induced refractive index change, in contrast to VNSE, which always gives the hybrid solitary wave solutions with a balanced contribution from the two transverse components irrespective of the induced index change. The analysis on the first higher-order vector solitary wave solution also indicates qualitative and quantitative differences in bifurcation characteristics based on the two sets of equations. However, fundamental differences occur for the second higher-order solitary wave solutions from the two sets of the equations-the triply degenerate solitary wave solutions resulting from Maxwell's equations cannot be recovered from VNSE even in the limit of small induced index change under which VNSE is derived. Finally we show that a general class of circularly polarized hybrid vector solitary waves with different propagation constants for the two transverse components obtained from VNSE does not satisfy (or has no counterpart from) Maxwell's equations in the limit of small induced refractive index change within which the two sets of equations are supposed to agree.

\section{GOVERNING EQUATIONS}

The propagation of a light beam in a medium is governed by Maxwell's equations, which involve the electric and magnetic fields $\mathbf{E}$ and $\mathbf{H}$. Expressing $\mathbf{H}$ in terms of $\mathbf{E}$, Maxwell's equation for a $\mathrm{cw}$ wave can be written equivalently in the form of the vector wave equation governing the electric field E:

$$
\boldsymbol{\nabla} \times \boldsymbol{\nabla} \times \mathbf{E}-k^{2} n^{2} \mathbf{E}-\left(k^{2} / \varepsilon_{0}\right) \mathbf{P}^{\mathrm{NL}}=\mathbf{0},
$$

where $k$ and $\varepsilon_{0}$ are the wave number and permittivity in free space, $n$ is the refractive index of a medium, which is a constant $n=n_{0}$ for a uniform medium to be considered, the nonlinear polarization $\mathbf{P}^{\mathrm{NL}}$ according to Maker et al. [23] for (linear) isotropic centrosymmetric materials has the form

$$
\mathbf{P}^{\mathrm{NL}}=A\left(\mathbf{E} \cdot \mathbf{E}^{*}\right) \mathbf{E}+0.5 B(\mathbf{E} \cdot \mathbf{E}) \mathbf{E}^{*},
$$

with $A$ and $B$ real nonlinear susceptibilities varying with materials, and $A>0$ and $B>0$ in a self-focusing nonlinear medium to be discussed. Dropping the term $\boldsymbol{\nabla \nabla} \cdot \mathbf{E}$ in $\boldsymbol{\nabla} \times \boldsymbol{\nabla} \times \mathbf{E}$ and assuming the slowly varying approximation [i.e., $\quad \partial^{2} \mathbf{E} / \partial z^{2}=\partial^{2} \mathbf{e} \exp \left(i k n_{0} z\right) / \partial z^{2} \approx i 2 k n_{0} \partial \mathbf{e} / \partial z-k^{2} n_{0}^{2} \mathbf{e}$ ], the vector wave equation (1) reduces to a VNSE [14]:

$$
i 2 k n_{0} \partial \mathbf{e} / \partial z+\nabla_{t}^{2} \mathbf{e}+\left(k^{2} / \varepsilon_{0}\right)\left[A\left(\mathbf{e} \cdot \mathbf{e}^{*}\right) \mathbf{e}+0.5 B(\mathbf{e} \cdot \mathbf{e}) \mathbf{e}^{*}\right]=0,
$$

where the transverse operator $\nabla_{t}^{2}=\nabla^{2}-\partial^{2} / \partial z^{2}$.

For the sake of convenience in illustrating the point, we consider, in the following, the $(1+1)$-dimensional case for which the stationary vector solitary wave solution to Eq. (1) is of the form [26]

$$
\mathbf{E}(x, z)=\left[E_{x}(x) \hat{\mathbf{x}}+\xi E_{y}(x) \hat{\mathbf{y}}+E_{z}(x) \hat{\mathbf{z}}\right] \exp (i \beta z),
$$

with $\xi=1$ corresponding to two transverse components being in phase, $\xi=i$ representing the solutions with the two transverse components out of phase by $\pi / 2$, and $\hat{\mathbf{x}}, \hat{\mathbf{y}}, \hat{\mathbf{z}}$ are unit vectors. This vector field solution of Eq. (3), substituted into Eq. (1), leads to the three coupled scalar equations governing real normalized field components $\Psi_{x, y}$ $=E_{x, y} \sqrt{(A+0.5 B) / \varepsilon_{0}} / b n_{0}$ and $\Psi_{z}=-i E_{z} \sqrt{(A+0.5 B) / \varepsilon_{0}} /$ $b n_{0}$ :

$$
\frac{d \psi_{x}}{d X}=b \sqrt{1+b^{2}} \frac{-2 \sigma \psi_{x}\left(\psi_{y} \psi_{y}^{\prime} / s+\psi_{z} \psi_{z}^{\prime}\right) b / \sqrt{1+b^{2}}+\left(\sigma \psi_{x}^{2}+s \psi_{y}^{2}+\psi_{z}^{2}+1 / b^{2}\right) \psi_{z}}{1+b^{2}\left[3 \psi_{x}^{2}+(\sigma / s) \psi_{y}^{2}+\sigma \psi_{z}^{2}\right]}
$$

$$
\begin{array}{r}
d^{2} \psi_{y} / d X^{2}=\psi_{y}-\left[(\sigma / s) \psi_{x}^{2}+\psi_{y}^{2}+s \psi_{z}^{2}\right] \psi_{y}, \\
d \psi_{z} / d X=b\left[1-\psi_{x}^{2}-(\sigma / s) \psi_{y}^{2}-\sigma \psi_{z}^{2}\right] \psi_{x} / \sqrt{1+b^{2}},
\end{array}
$$

with $\quad X=k n_{0} b x, \quad b=\sqrt{\beta^{2} / k^{2} n_{0}^{2}-1}, \quad \sigma=(A-0.5 B) /$ $(A+0.5 B)$ (which is 0.075 for $0.5 B / A=0.86$ of carbon disulphide liquid and $\sigma=1 / 3$ for $0.5 B / A=0.5$ of some crystals $[5,23])$, the prime indicating the derivative with respect to $X$, and $s=1$ for $\xi=i$ and $s=\sigma$ for $\xi=1$. Following similar substitution, the corresponding normalized nonlinear Schrödinger equation is written as

$$
\begin{aligned}
& d^{2} \psi_{x} / d X^{2}=\left[1-\psi_{x}^{2}-(\sigma / s) \psi_{y}^{2}\right] \psi_{x}, \\
& d^{2} \psi_{y} / d X^{2}=\left[1-(\sigma / s) \psi_{x}^{2}-\psi_{y}^{2}\right] \psi_{y},
\end{aligned}
$$

with $\mathbf{e}$ and its components related by $\mathbf{e}(x, z)=\left[E_{x}(x) \hat{\mathbf{x}}\right.$ $\left.+\xi E_{y}(x) \hat{\mathbf{y}}\right] \exp \left[i\left(\beta^{2}-k^{2} n_{0}^{2}\right) z / 2 k n_{0}\right]$. In the special case of $\psi_{x}=0\left(\psi_{z}=0\right)$, Eqs. (4) and (5) reduce to the same equation governing the field $\psi_{y}$ of the TE type spatial solitary wave, the solution of which is described by sech function $[1,8]$.
Another special case is $\psi_{y}=0$; Eq. (4) gives the TM type solitary wave solution, which is quantitatively different from that predicted by Eq. (5). The difference increases with increasing $b$ [27].

\section{FUNDAMENTAL SOLITARY WAVE STATES}

\section{A. Difference}

Now we consider general cases of $\psi_{x} \neq 0$ and $\psi_{y} \neq 0$ where many interesting solitary wave states arise. For the case of $s / \sigma=1$ (i.e., $\xi=1$ for two in phase transverse components), Eq. (5) is shown to be integrable [14], and supports a class of linearly polarized fundamental vector solitary waves with the polarization orienting in an arbitrarily direction,

$$
\begin{aligned}
& \psi_{x}=\cos \theta \sqrt{2} \operatorname{sech}\left(X-X_{0}\right) \\
& \psi_{y}=\sin \theta \sqrt{2} \operatorname{sech}\left(X-X_{0}\right),
\end{aligned}
$$

where $\theta$ refers to the polarization direction. This family of solitary wave solutions to the VNSE seems to indicate that 
there is a corresponding family of linearly polarized hybrid TE-TM trapped vector solitary waves to Maxwell's equations (4) transiting continuously from the special TE solitary wave to the TM solitary wave. Our analysis on Eq. (4) for this case reveals that the vector solitary waves of this type do not exist for any $b$ except for the special TE solitary wave $(\theta$ is an odd integer of $\pi / 2$ ) or for the special TM solitary wave ( $\theta$ is an integer of $\pi$ ). Physically, the existence of a linearly polarized solitary wave orienting in any direction to the VNSE is due to the rotational symmetry of the induced waveguide (i.e., the linearly polarized solitary wave does not "see" the refractive index change particularly aligned in the $x$ direction). However, the possible solitary wave state to Maxwell's equations "sees" the induced waveguide especially along the $x$ direction, imposing the effect that varies with the orientation of the polarization on the solitary wave beam. That is, the rotational symmetry present in the VNSE is broken in Maxwell's equations; self-consistency of selftrapping of the linearly polarized TE-TM vector solitary wave no longer holds for Maxwell's equations [28]. Mathematically, the difference in the existence of the linear polarized hybrid TE-TM vector solitary waves results from the difference in the integrability of the systems. Equation (5) at $s / \sigma=1$ possesses two integrals of motion; one is the Hamiltonian

$$
H=\psi_{x}^{\prime 2}+\psi_{y}^{\prime 2}-\psi_{x}^{2}-\psi_{y}^{2}+0.5\left(\psi_{x}^{4}+\psi_{y}^{4}\right)+(\sigma / s) \psi_{x}^{2} \psi_{y}^{2}
$$

the other is $\psi_{x} \psi_{y}^{\prime}-\psi_{x}^{\prime} \psi_{y}=$ const, and Eq. (5) is integrable. However, Eq. (4) admits only one invariant,

$$
\begin{aligned}
H= & \psi_{y}^{\prime 2}+\frac{b^{2}}{1+b^{2}}\left[1-\psi_{x}^{2}-\frac{\sigma}{s} \psi_{y}^{2}-\sigma \psi_{z}^{2}\right]^{2} \psi_{x}^{2}-\psi_{x}^{2}-\psi_{y}^{2}+\frac{\psi_{z}^{2}}{b^{2}} \\
& +\frac{1}{2}\left(\psi_{x}^{4}+\psi_{y}^{4}+\psi_{z}^{4}\right)+s \psi_{y}^{2} \psi_{z}^{2}+\sigma \psi_{x}^{2}\left(\frac{\psi_{y}^{2}}{s}+\psi_{z}^{2}\right),
\end{aligned}
$$

regardless the value of $s / \sigma$ and it is not integrable. The linearly polarized solitary wave solutions are lost when the system transfers from integrable to nonintegrable.

\section{B. Similarity}

On the other hand, for the general case of $s / \sigma \neq 1$, the induced refractive index is anisotropic in nature; the hybrid fundamental TE-TM vector solitary waves are found to exist in Maxwell's equations. These solutions correspond to the trajectory of Eq. (8) with $H=0$. A fixed anisotropy $\sigma(\neq 1)$ and induced index change $\left(\Delta n \sim b^{2}=\beta^{2} / k^{2} n_{0}^{2}-1\right)$ leads to a hybrid vector solitary wave with the field values at $X=0$ related by

$$
\begin{aligned}
& b^{2}\left[1-\psi_{x}^{2}-(\sigma / s) \psi_{y}^{2}\right]^{2} \psi_{x}^{2} /\left(1+b^{2}\right)-\psi_{x}^{2}-\psi_{y}^{2}+0.5\left(\psi_{x}^{4}+\psi_{y}^{4}\right) \\
& \quad+\sigma \psi_{x}^{2} \psi_{y}^{2} / s=0,
\end{aligned}
$$

which reduces to the corresponding one for VNSE (5) by setting $b=0$. Note that for $s / \sigma \neq 1$, Eq. (5) is not integrable either and it admits one invariant of Eq. (7). The corresponding fundamental vector solitary wave solutions to Eq. (5) read $\psi_{x}=\psi_{y}=\sqrt{2 /(1+\sigma)} \operatorname{sech}\left(X-X_{0}\right)$ identified by the straight (dashed) line, independent of $b$, on the plane $\psi_{z}=0$

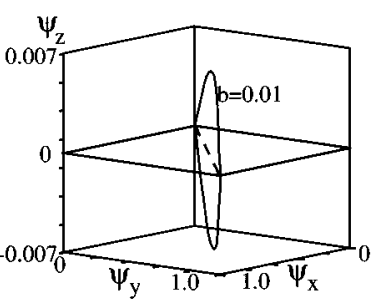

(a)

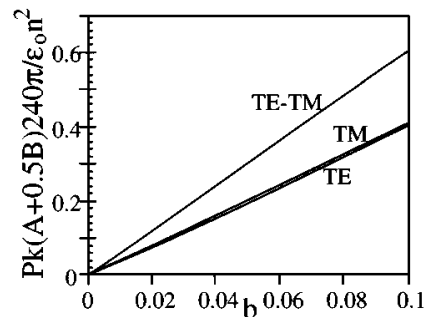

(b)
FIG. 1. (a) Phase space trajectories of the fundamental vector solitons for $b=0.01$ (solid curve) and the dashed line is the corresponding trajectory from VNSE; (b) dispersion curve describing the dependence of power on $b$ or the propagation constant $\beta$ of TE-TM vector solitons. The value $\sigma=1 / 3$ and $s=1$ in the figures.

in Fig. 1(a) of three-dimensional phase space trajectory. The solitary wave solutions for $b \neq 0$ (or $\beta>k n_{0}$ ) from Maxwell's equations are, however, represented by $3 \mathrm{D}$ curved trajectories [see Fig. 1(a) for the example of $b=0.01$ and $\sigma=1 / 3$ ]. With increasing $b$ (or $\beta$ ) $\psi_{x}$ and $\psi_{z}$ (TM) components intensify while $\psi_{y}$ (TE) component diminishes. The power carried by the hybrid TE-TM vector solitary wave

$$
\begin{aligned}
P= & \frac{1}{2} \int_{-\infty}^{\infty} \operatorname{Re}\left(\mathbf{E} \times \mathbf{H}^{*}\right) \cdot \mathbf{z} d x \\
= & \frac{\beta \varepsilon_{0} b n_{0}}{240 \pi k^{2}(A+0.5 B)} \int_{-\infty}^{\infty}\left\{\left|\psi_{x}\right|^{2}+\left|\psi_{y}\right|^{2}+\frac{b^{2}\left|\psi_{x}\right|^{2}}{1+b^{2}}\right. \\
& \left.\times\left[\left|\psi_{x}\right|^{2}+(\sigma / s)\left|\psi_{y}\right|^{2}+\sigma\left|\psi_{z}\right|^{2}-1\right]\right\} d X
\end{aligned}
$$

is plotted in Fig. 1(b) together with those of TE and TM solitary waves for $\sigma=1 / 3$. Other values of $\sigma$ demonstrate similar characteristics. The hybrid solitary wave has the largest amount of power.

Here, the characteristics of the solitary wave solutions depend apparently on the parameter $b$, which is related to the beam width $a_{0}$ in experiment [5] by $b=\sqrt{\beta^{2} / k^{2} n_{0}^{2}-1}$ $=1 / k n_{0} a_{0}$. The beam width $a_{0}=8.5 \mu \mathrm{m}$ at power $230 \mathrm{~kW}$ in the experiment [5] for nonlinear glass gives $b=0.0067$, corresponding to the nonlinear induced index change $\Delta n$ $=n_{\max }-n_{0} \sim \beta / k-n_{0} \approx\left(\beta / k-n_{0}\right)\left(\beta / k+n_{0}\right) / 2 n_{0}=b^{2} n_{0} / 2$ $=4.4 \times 10^{-5}$. On the other hand, when the power increases to $400 \mathrm{~kW}$ [Fig. 2(c) of Ref. [5]], the beam width becomes $a_{0}=4.9 \mu \mathrm{m}$, which gives $b=0.0132$ and $\Delta n=1.33 \times 10^{-4}$. Obviously, the value $b=0.01$ (corresponding to $\Delta n=7.6 \times 10^{-5}$ ) of Fig. 1(a) is well within the present experimental condition in a glass medium [5] with weak nonlinear coefficient $n_{2}=A /\left(2 n_{0} \varepsilon_{0}\right)=3.4 \times 10^{-16} \mathrm{~cm}^{2} / \mathrm{W}$. In fact, for nonlinear materials such as $\mathrm{CS}_{2}$ [4] and AlGaAs, which has a nonlinear coefficient [6] $n_{2}=5.4 \times 10^{-14} \mathrm{~cm}^{2} / \mathrm{W}$ 160 times larger than in glass, the values of $b$ and $\Delta n$ can possibly reach $b \sim 0.1$ and $\Delta n \sim 0.01$ or higher (as $b^{2} \sim \Delta n \sim n_{2} \times$ intensity).

\section{FIRST HIGHER-ORDER SOLITARY WAVE STATES}

Apart from the fundamental solitary wave solutions considered above, system (4) also supports higher-order solitary 


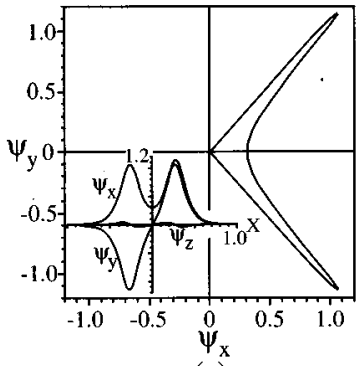

(a)

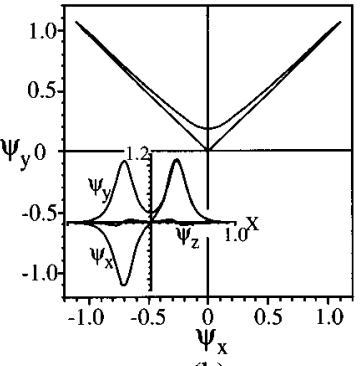

(b)
FIG. 2. Projection of phase space trajectories on $\psi_{z}=0$ plane and field profiles of the first higher-order vector solitons at $\sigma=2 / 3, s=1$, and $b=0.06$, which corresponds to the nonlinearity induced refractive index change $\Delta n \approx b^{2} n_{0} / 2=0.0027$ for $n_{0}=1.53$.

wave solutions. The first higher order is characterized by two humps in the total intensity $|\mathbf{E}|^{2}$ profile symmetrically located on the two sides of $x=0$ at which the fields have the characteristics of (i) $\psi_{y}(0)=0, \psi_{z}(0)=0$, and

$$
\psi_{y}^{\prime}(0)=\psi_{x}(0)\left\{1-0.5 \psi_{x}^{2}(0)-b^{2}\left[1-\psi_{x}^{2}(0)\right]^{2} /\left(1+b^{2}\right)\right\}^{1 / 2},
$$

and (ii) $\psi_{x}(0)=0, \psi_{y}^{\prime}(0)=0$ and

$$
\begin{aligned}
\psi_{z}^{2}(0)= & -s \psi_{y}^{2}(0)-1 / b^{2} \\
& +\sqrt{1 / b^{4}+2\left(1+s / b^{2}\right) \psi_{y}^{2}(0)-0.5\left(1-s^{2}\right) \psi_{y}^{4}(0)},
\end{aligned}
$$

corresponding to two degenerate solitary wave states [Figs. 2(a) and 2(b)] associated with a particular $b$ (or $\beta$ ) below a critical value $b=b_{c 1}$. This critical value $b_{c 1}$, above which the degenerate solitary wave specified by Eq. (10b) ceases to exist or each $b$ corresponds to one solitary wave state only, decreases with increasing $\sigma$ and $b_{c 1} \rightarrow 0$ when $\sigma>0.8(B$ $<0.22 \mathrm{~A})$ as shown in Fig. 3(a). The difference in the field shapes and the phase space trajectories between the degenerate solitary waves also increases with increasing $b$. On the other hand, VNSE (5) invariably yields two degenerate first higher-order solitary wave solutions (related by the transfor-

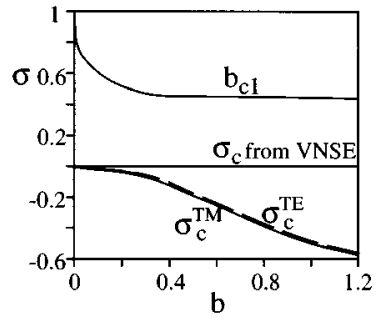

(a)

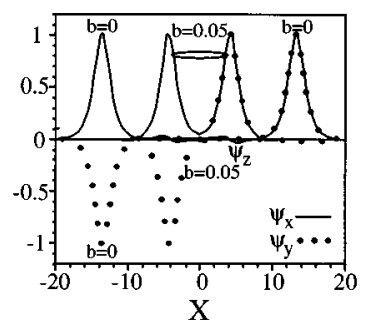

(b)
FIG. 3. (a) The function $b_{c 1}(\sigma)$ vs $\sigma$, and the dependence of the bifurcation values $\sigma_{c}^{\mathrm{TM}}$ and $\sigma_{c}^{\mathrm{TE}}$ on the waveguide height $b$ obtained from Maxwell's equations; $\sigma_{c}$ is the corresponding one from VNSE. (b) Field profiles of the degenerate second-order vector soliton [with the fields at $x=0$ governed by Eq. (10a)] at $\sigma=0.94$ and $s=1$ for $b=0.05$ and $b=0$ (from VNSE), respectively; note that at this $b=0.05$, according to Maxwell's equation the other degenerate second-order vector soliton with the fields at $x=0$ governed by Eq. (10b) does not exist although VNSE still predicts its existence.

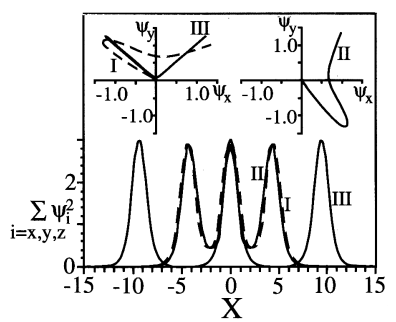

(a)

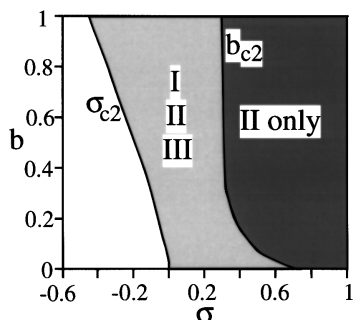

(b)
FIG. 4. (a) The intensity distributions of three degenerate thirdorder vector solitons at $\sigma=1 / 3, s=1$, and $b=0.05$ obtained from Maxwell's equations, and the corresponding two degenerate solitons from the VNSE have the same intensity profiles distributing between degenerate solitons I and II intensity profiles in the figure. (b) Bifurcation values $\sigma_{c 2}(b)$ vs $b$ and $b_{c 2}(\sigma)$ vs $\sigma$.

mation $\psi_{x, y} \leftrightarrow \psi_{y, x}$ or rotating phase space trajectory by $90^{\circ}$ [see Eq. (5)]), irrespective of the value of $b$; and they exist within $\left(\sigma_{c}=\right) 0<\sigma<1$ (or $2 \mathrm{~A}>B>0$ ), meaning that within $1>\sigma>0.8$ Maxwell's equations differ from the VNSE qualitatively even in the limit of $b \rightarrow 0$. Furthermore, for the value $\sigma<0$ (or $B>2$ A) Maxwell's equations (4) also admit this set of higher-order solutions yet VNSE accepts no such solutions when $\sigma<0$. The relation between $b$ and the bifurcation values $\sigma=\sigma_{c}^{\mathrm{TM}}(<0)$ and $\sigma=\sigma_{c}^{\mathrm{TE}}(<0)$, at which the degenerate first higher-order solitary waves [specified by Eqs. (10a) and (10b)] bifurcate from TM and TE solitary waves, is shown in Fig. 3(a).

The interesting aspect of this vector solitary wave is that a superposition of $\psi_{x}$ and $\psi_{y}$ components (at $b \rightarrow 0$ ) may lead to two parallel propagating "fundamental solitary waves", with their polarizations orthogonal to each other. This can be appreciated by considering a case of $\sigma=0.94(B=0.062 \mathrm{~A})$ for which the field of $\psi_{x}$ and $\psi_{y}$ can be approximated by $\psi_{x}=\psi_{1}+\psi_{2}=\psi\left(X-X_{0}\right)+\psi\left(X+X_{0}\right) \quad$ and $\quad \psi_{y}=\psi_{1}-\psi_{2}$ $=\psi\left(X-X_{0}\right)-\psi\left(X+X_{0}\right)$ with $\psi(\zeta) \cong \operatorname{sech}(\zeta) / \sqrt{2}$ [Fig. 3(b)]. For $\xi=i$, the superposition leads to two parallel propagating solitary waves with one left-circularly polarized and the other right-circularly polarized as $\mathbf{E} \exp (-i \beta z) \sim\left(\psi_{1}+\psi_{2}\right) \mathbf{x}$ $+i\left(\psi_{1}-\psi_{2}\right) \mathbf{y}+i \psi_{z} \mathbf{z}=(\mathbf{x}+i \mathbf{y}) \psi_{1}+(\mathbf{x}-i \mathbf{y}) \psi_{2}+i \psi_{z} \mathbf{z} \quad\left(\psi_{z} \rightarrow 0\right.$ at $b \rightarrow 0$ ); and for $\xi=1$ two linearly polarized "fundamental solitary waves" result from the superposition because $\mathbf{E} \exp (i \beta z) \sim\left(\psi_{1}+\psi_{2}\right) \mathbf{x}+\left(\psi_{1}-\psi_{2}\right) \mathbf{y}+i \psi_{z} \mathbf{z}=(\mathbf{x}+\mathbf{y}) \psi_{1}+(\mathbf{x}$ $-\mathbf{y}) \psi_{2}+i \psi_{z} \mathbf{z}$. The normalized separation $2 X_{0}$ from the VNSE is fixed irrespective of $b$, which is $2 X_{0}=26.8$ for $\sigma=0.94$ [Fig. 3(b)]. However, Maxwell's equations give the normalized separation decreasing with increasing $b$, which is $2 X_{0}=8.3$ for $b=0.05$ (corresponding to $\left.\Delta n \approx b^{2} n_{0} / 2=0.0019\right)$, over three times smaller than that predicted by Eq. (5) [Fig. 3(b)].

\section{SECOND- AND OTHER HIGHER-ORDER SOLITARY WAVE STATES}

The next higher-order vector solitary wave features three peaks in the total $|\mathbf{E}|^{2}$ intensity profile [Fig. 4(a)]. The relation of the field components at $x=0$ for this vector solitary wave is governed by Eq. (9). This set of higher-order vector solitary waves bifurcates from the TE and TM solitary waves at $\sigma_{c 2}(b)[<0$, see Fig. $4(b)]$ and continues to exist up to $\sigma=1$. Within $\sigma_{c 2}<\sigma<1$, Eq. (4) yields three degenerate 
third-order vector solitary waves for each $b$ (or $\beta$ ) below a particular $b=b_{c 2}(\sigma)$ (or $k n_{0}<\beta<\beta_{c}$ ) as is shown in Fig. 4(a) for the case of $\sigma=1 / 3$ and $b=0.05$. This critical $b_{c 2}(\sigma)$ confining $b$ for trapping three degenerate third-order vector solitary waves depends on $\sigma$ [see Fig. 4(b)]. It decreases with increasing $\sigma$ and at $\sigma>0.7(B<0.35 \mathrm{~A}) \quad b_{c 2}(\sigma) \rightarrow 0$. At $b=b_{c 2}(\sigma)$ the two degenerate solitary waves (I and III in Fig. 4) merge into one, and above $b>b_{c 2}(\sigma)$ the two degenerate solitary waves disappear and each $b$ (or $\beta$ ) is associated with only one third-order vector solitary wave. On the contrary, VNSE (5) predicts that each $b$ corresponds to two degenerate vector solitary waves (related by transformation $\psi_{x, y} \leftrightarrow \psi_{x, y}$ ) for any value $b>0$ that exists within $0<\sigma<1$, demonstrating a qualitative difference from Maxwell's equations.

Furthermore, it can be shown that Maxwell's equations admit fourth-, fifth-, and other higher-order vector solitary waves that exhibit qualitatively different bifurcation characteristics from those acquired from the VNSE. Instead of such continuation, we conclude our discussion here by pointing out another fundamental difference between Maxwell's equations and the VNSE in predicting vector solitary wave solutions with circularly polarizations.

\section{CIRCULARLY POLARIZED SOLITARY WAVE STATES}

It can be shown that the VNSE supports a general class of vector solitary waves with different propagation constants for two circularly polarized transverse components. Expressing the transverse field component $\mathbf{e}_{t}$ as left and right circular polarizations $\quad \mathbf{e}_{t}=\left(e_{r}+e_{1}\right) / \sqrt{2} \mathbf{x}+i\left(e_{r}-e_{1}\right) / \sqrt{2} \mathbf{y}=e_{r}(\mathbf{x}+i \mathbf{y}) /$ $\sqrt{2}+e_{1}(\mathbf{x}-i \mathbf{y}) / \sqrt{2}=e_{+}(x) \exp \left[i\left(\beta_{+}^{2}-k^{2} n_{0}^{2}\right) z / 2 k n_{0}\right] \mathbf{r}+$ $e_{-}(x) \exp \left[i\left(\beta_{-}^{2}-k^{2} n_{0}^{2}\right) z / 2 k n_{0}\right] \mathbf{l}$, Eq. (2) reads

$$
d^{2} e_{ \pm} / d x^{2}+\left[A\left|e_{ \pm}\right|^{2}+(A+B)\left|e_{\mp}\right|^{2}\right] e_{ \pm}=\left(\beta_{ \pm}^{2}-k^{2} n_{0}^{2}\right) e_{ \pm},
$$

which admits the fundamental and higher-order circularly polarized solitary waves for $\beta_{+} \neq \beta_{-}$[18]. However, Maxwell's equations do not accept this kind of vector solitary wave solutions. This can be illustrated by expanding Eq. (1) in terms of $e_{ \pm}\left[\mathbf{E}_{t}=\mathbf{E}-E_{z} \mathbf{z}=e_{+}(x) \exp \left(i \beta_{+} z\right) \mathbf{r}\right.$ $\left.+e_{-}(x) \exp \left(i \beta_{-} z\right) l\right]$, leading to

$$
\begin{gathered}
\frac{d^{2}\left[e_{ \pm}-e_{\mp} e^{i\left[ \pm\left(\beta_{-}-\beta_{+}\right) z\right]}\right]}{2 d x^{2}}+\left[A\left(\left|e_{ \pm}\right|^{2}+\left|e_{z}\right|^{2}\right)\right. \\
\left.+(A+B)\left|e_{\mp}\right|^{2}\right] e_{ \pm}+0.5 B e_{z}^{2} e_{+}^{*} \eta_{-} \\
=\left(\beta_{ \pm}^{2}-k^{2} n_{0}^{2}\right) e_{ \pm}+\eta_{ \pm} \frac{i \beta_{+}}{\sqrt{2}} \frac{d e_{z}}{d x}
\end{gathered}
$$

which reduces to Eq. (11) when $e_{z}=0 \quad(b=0)$ and $-i \beta_{+} \sqrt{2} d e_{z} / d x=d^{2}\left\{e_{+}+e_{-} \exp \left[i\left(\beta_{-}-\beta_{+}\right) z\right]\right\} / d x^{2}$, where $\eta_{+}=1$ and $\eta_{-}=\exp \left[i\left(\beta_{+}-\beta_{-}\right) z\right]$. Obviously, no stationary solutions exist to Eq. (12) for any values of $A$ and $B(\neq 0)$ except for $\beta_{+}=\beta_{-}$.

\section{DISCUSSION}

Here, it should be mentioned that the physical origin of fundamental differences demonstrated above in the vector solitary wave solutions predicted by the two sets of equations within the parameter region where the approximation is valid result from the self-consistency requirement for selftrapping. In some cases for which fundamental differences occur, the presence of the longitudinal field, no matter how small it is, leads to anisotropy in the induced refractive index change, which destroys the self-consistency for self-trapping. This is contrary to the linear guiding structure where the longitudinal component can always be ignored safely in the weakly guiding approximation [29].

It should also be added that the discussion concerning the similarity and difference between Maxwell's equations and the VNSE presented above has been restricted to the bright stationary solitary wave solutions. Applications of these stationary solitary wave solutions for a long or short distance propagation requires the knowledge of their stability characteristics. Stable solitary wave states ensure a long distance evolution of the stationary structure, while unstable solitary wave states may find their way in applications for ultrafast optical switching [30]. The stability analysis of these stationary solitary wave states and the corresponding differences between the two sets of equations are beyond the scope of the present paper and require further investigation.

Equally, mention should be made that the analysis on similarities and differences between the VNSE and Maxwell's equations for predicting the solitary wave solutions presented are for the two transverse components being either in phase or $\pi / 2$. The corresponding $E_{x}$ and $E_{y}$ for the solitary wave solutions of Eqs. (4) and (5) are then the real quantities [31]. When the relative phase between the two transverse components is neither $m \pi$ nor $\pi / 2+m \pi(m=0,1,2, \ldots)$ and/or the phases of amplitudes are the transverse dimension (such as $x$ ) dependent, the field components $E_{x}$ and $E_{y}$ of possible solitary solutions become complex. The VNSE then involves four real equations [rather than two of Eqs. (5)] governing the real and imaginary parts of the two transverse components for solitary wave solutions. The corresponding Maxwell's equations then include six real equations [rather than three of Eqs. (4)] governing the real and imaginary parts of the three field components. Vector dark solitons in a selfdefocusing nonlinear medium are such examples with complex $E_{x}, E_{y}$, and $E_{z}$. In the moving coordinates $(X, Y, Z)$ transformed from $(X=x-z / v, Y=y, Z=z)$, these vector dark solitons are stationary with respect to the propagation distance, i.e., $E_{x, y, z}(X, Y)$ are $Z$ independent. The phases of amplitudes of the solitons are transverse dimension (such as $X$ ) dependent and so is the relative phase. In these general situations, similarities and differences between the VNSE and Maxwell's equations for predicting solitary wave solutions are still expected (namely some solutions of the VNSE can be recovered from Maxwell's equations, others are not, and vice versa), although details may differ from case to case. These elaborations are again beyond the scope of the present paper and require further investigation. However, the main conclusions drawn from the bright solitary waves for the relative phase being 0 or $\pi / 2$ still apply; i.e., Maxwell's equations have to be used for describing light evolution in a 
nonlinearity induced anisotropic medium when the polarization characteristics of a solitary wave is considered.

\section{CONCLUSION}

In summary, similarities and fundamental differences between Maxwell's equations and the nonlinear Schrödinger equation are examined. Our study shows that Maxwell's equations have to be used for describing light evolution in a nonlinearity induced anisotropic medium when the polarization characteristics of a solitary wave are considered, especially for the higher-order solitary wave states. In particular, it is shown that arbitrarily linearly polarized vector solitary waves predicted by VNSE do not satisfy Maxwell's equations even in the limit of small induced refractive index change under which VNSE is derived. The existence of novel classes of hybrid TE-TM vector solitary waves is also predicted and the bifurcation characteristics are investigated.

\section{ACKNOWLEDGMENTS}

The optical sciences centre is a member of the Australian Photonics Research Centre, and Y. Chen is grateful for the financial support of the Australian Research Council.
[1] R. Y. Chiao, E. Garmire, and C. H. Townes, Phys. Rev. Lett. 13, 479 (1964).

[2] H. A. Haus, Appl. Phys. Lett. 8, 128 (1966).

[3] Y. Chen, Opt. Lett. 16, 4 (1991).

[4] A. Barthelemy, S. Maneuf, and C. Froehly, Opt. Commun. 55, 201 (1985); S. Maneuf, R. Desailly, and C. Froehly, Opt. Commun. 65, 193 (1988); S. Maneuf and F. Reyneud, ibid. 66, 325 (1988).

[5] J. S. Aitchison, A. M. Weiner, Y. Silberberg, M. K. Oliver, J. L. Jackel, D. E. Leaird, E. M. Vogel, and P. W. Smith, Opt. Lett. 15, 471 (1990); J. S. Aitchison, Y. Silberberg, A. M. Weiner, D. E. Leaird, M. K. Oliver, J. L. Jackel, E. M. Vogel, and P. W. Smith, J. Opt. Soc. Am. B 8, 1290 (1991).

[6] J. S. Aitchison, K. Al-Hemyari, C. N. Ironside, R. S. Grant, and W. Sibbett, Electron. Lett. 28, 1879 (1992).

[7] G. A. Swartzlander, D. R. Anderson, J. J. Regan, H. Yin, and A. E. Kaplan, Phys. Rev. Lett. 66, 1583 (1991); B. LutherDavies and X. Yang, Opt. Lett. 17, 496 (1992); 17, 1755 (1992).

[8] V. E. Zakharov and A. B. Shabat, Zh. Eksp. Teor. Fiz. 61, 118 (1971) [Sov. Phys. JETP 34, 62 (1972)].

[9] P. L. Kelley, Phys. Rev. Lett. 15, 1005 (1965).

[10] S. A. Akhmanov, R. V. Khokhlov, and A. P. Sukhorukov, in Laser Handbook, edited by F. T. Arecchi and E. O. SchulzDuBois (North-Holland, Amsterdam, 1972), p. 1151, and references therein.

[11] E. A. Kuznetsov and S. K. Turitsyn, Zh. Eksp. Teor. Fiz. 94, 119 (1988) [Sov. Phys. JETP 67, 1583 (1988)].

[12] G. A. Swartzlander and C. T. Law, Phys. Rev. Lett. 69, 2503 (1992)

[13] A. E. Kaplan, Phys. Rev. Lett. 55, 1291 (1985).

[14] S. V. Manakov, Zh. Eksp. Teor. Fiz. 65, 505 (1973) [Sov. Phys. JETP 38, 248 (1974)].

[15] M. V. Tratnik and J. E. Sipe, Phys. Rev. A 38, 2011 (1988).

[16] D. N. Christodoulides and R. I. Joseph, Opt. Lett. 13, 53 (1988).

[17] C. R. Menyuk, J. Opt. Soc. Am. B 5, 392 (1988).

[18] V. M. Eleonskii, V. G. Korolev, N. E. Kulagin, and L. P. Shil'nikov, Zh. Eksp. Teor. Fiz. 99, 1113 (1991) [Sov. Phys. JETP 72, 619 (1991)]; N. N. Akhmediev, A V. Buryak, J. M. Soto-Crespo, and D. R. Andersen, J. Opt. Soc. Am. B 12, 434 (1995)
[19] M. Haelterman, A. P. Sheppard, and A. W. Snyder, Opt. Lett. 18, 1406 (1993).

[20] Y. Chen and J. Atai, Phys. Rev. E 52, 3102 (1995).

[21] A. W. Snyder, S. J. Hewlett, and D. J. Mitchell, Phys. Rev. Lett. 72, 1012 (1994).

[22] Y. Silberberg and Y. Barad, Opt. Lett. 20, 246 (1995).

[23] P. D. Maker, R. W. Terhune, and C. M. Savage, Phys. Rev. Lett. 12, 507 (1964).

[24] A. W. Snyder, D. J. Mitchell, and Y. Chen, Opt. Lett. 19, 524 (1994).

[25] V. M. Eleonskii, L. G. Oganes'yants, and V. P. Silin, Zh. Eksp. Teor. Fiz. 63, 532 (1972) [Sov. Phys. JETP 36, 282 (1973)].

[26] There is no dynamic solitary wave with different propagation constants $\beta_{x}$ and $\beta_{y}$ for the two transverse field components in a nonlinear $B \neq 0$ medium considered here. This is in contrast to those reported in Refs. [24] for the nonlinear $B=0$ medium.

[27] Y. Chen and A. W. Snyder, Electron. Lett. 27, 565 (1991); Y. Chen, ibid. 27, 380 (1991).

[28] A question may arise here as to what will happen when a Manakov solitary wave linearly polarized, (e.g., at $45^{\circ}$ ) is initially launched. This initial excitation will certainly not be selftrapped. Rather, the excitation of Eq. (6) will lead to coupling between TE and TM field components, which after a transit distance evolve to the stationary propagation of the TE solitary wave or TM solitary wave or hybrid TE-TM solitary wave with TE and TM components $\pi / 2$ out of phase (i.e., $s / \sigma \neq 1$ to be discussed subsequently), depending on the polarization of the initial beam. For the polarization around $45^{\circ}$, the beam will evolve to the hybrid TE-TM solitary wave and the initial excitation with the polarization around $90^{\circ}$ or $0^{\circ}$ will develop to TE or TM solitary wave.

[29] A. W. Snyder and J. D. Love, Optical Waveguide Theory (Chapman and Hall, New York, 1983).

[30] Y. Chen, Phys. Rev. E 50, 5205 (1994).

[31] The reason for the $z$ component chosen to be $\pm \pi / 2$ out of phase with the $x$ component is to recover the solutions of VNSE with real $E_{x}$ and $E_{y}$ from Maxwell's equations. If the $z$ component is not chosen in this way, the solutions to VNSE with real $E_{x}$ and $E_{y}$ considered in the paper are no longer recoverable from Maxwell's equations because Eqs. (4) then become complex and consequently $E_{x}$ and $E_{y}$ become complex. 